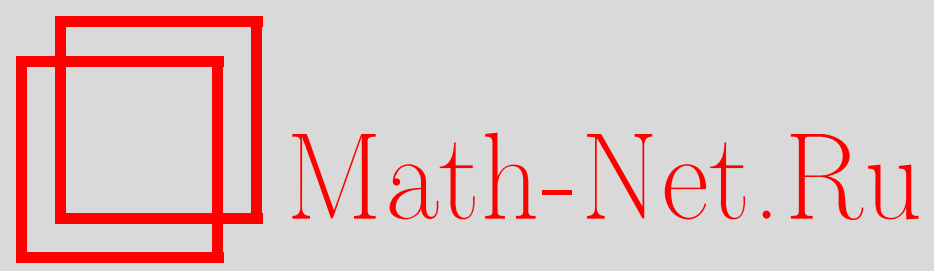

В. Л. Лясковский, И. Б. Бреслер, М. А. Алашеев, Формализация, оценка алгоритмической сложности и метод решения задачи выбора системотехнических решений по созданию (развитию) распределенных информационноуправляющих систем, Вестник ТвГУ. Серия: Прикладная математика, 2017, выпуск 3, 73-91

DOI: https://doi.org/10.26456/vtpmk180

Использование Общероссийского математического портала Math-Net.Ru подразумевает, что вы прочитали и согласны с пользовательским соглашением http://www . mathnet.ru/rus/agreement

Параметры загрузки:

IP: 34.227 .88 .159

26 апреля 2023 г., 10:11:42 
УДК 519.2

\section{ФОРМАЛИЗАЦИЯ, ОЦЕНКА АЛГОРИТМИЧЕСКОЙ СЛОЖНОСТИ И МЕТОД РЕШЕНИЯ ЗАДАЧИ ВЫБОРА СИСТЕМОТЕХНИЧЕСКИХ РЕШЕНИЙ ПО СОЗДАНИЮ (РАЗВИТИЮ) РАСПРЕДЕЛЕННЫХ ИНФОРМАЦИОННО-УПРАВЛЯЮЩИХ СИСТЕМ}

Лясковский В.Л., Бреслер И.Б., Алашеев М.А.

Научно-исследовательский институт информационных технологий, г. Тверь

Поступила в редакцию 24.05.2017, после переработки 12.07.2017.

В статье рассматривается постановка задачи выбора системотехнических решений по созданию (развитию) распределенных информационно-управляющих систем. Необходимость решения данной задачи обусловлена тем, что многие автоматизированные системы обработки информации и управления создаются и эксплуатируются в течение десятилетий, при этом в процессе эксплуатации меняются требования, предъявляемые к этим системам. Поэтому возникает необходимость в периодическом формировании решений по приведению состояния информационно-управляющей системы в соответствие новым требованиям. В качестве основного показателя эффективности формируемых решений принят комплексный показатель, характеризующий степень автоматизации функциональных процессов, реализуемых в системе. В качестве ограничений выступают требования к обязательной автоматизации наиболее важных функциональных процессов и своевременности их выполнения, а также предельно допустимые финансовые и временные ресурсы процесса развития системы. Проведенный анализ алгоритмической сложности решения задачи показывает невозможность ее решения путем рассмотрения всех возможных вариантов из-за экспоненциальной зависимости количества решений от размерности исходных данных. В связи с этим разработан эвристический алгоритм, основанный на использовании «жадных» методов оптимизации, позволяющий существенно сократить количество рассматриваемых вариантов и получить рациональное решение задачи при относительно небольшой вычислительной сложности.

Ключевые слова: система обработки информации и управления, проектирование автоматизированных систем управления, жизненный цикл систем управления, комплекс средств автоматизации.

Вестник ТвГУ. Серия: Прикладная математика. 2017. № 3. С. 73-91. https://doi.org/10.26456/vtpmk180

\section{Введение}

Управленческие решения по руководству министерствами, ведомствами РФ, 
государственными корпорациями, интегрированными структурами и предприятиями промышленности реализуются в системах обработки информации и управления (СОИУ), специально создаваемых в соответствующих министерствах, ведомствах и иных организациях РФ. Одним из основных путей повышения эффективности применения таких систем является комплексная автоматизация процессов обработки информации и управления, реализуемая в процессе создания (развития) соответствующих распределенных информационно-управляющих систем (РИУС).

РИУС - это распределенные автоматизированные СОИУ, создаваемые для оснащения ими органов управления соответствующих министерств, ведомств, агентств и иных организаций РФ в целях повышения эффективности применения управляемых органов (объектов). РИУС, как правило, представляет собой многоуровневую систему, состоящую из нескольких функциональных подсистем (ФПс), каждая из которых реализует ряд взаимоувязанных функциональных процессов (ФП). При этом отдельные ФП реализуются посредством выполнения комплексов функциональных задач ( $\Phi 3)$, решаемых в системе на различных уровнях управления СОИУ.

\section{1. Описание типовой структурной схемы РИУС}

Типовая структурная схема РИУС как объекта исследования приведена на Рис. 1 и включает в свой состав подсистему обработки информации и управления, подсистему передачи информации и подсистему внешних объектов [1]. При этом, подсистема обработки информации и управления РИУС состоит из органов управления (ОУ), которые, как правило, включают в свой состав центр обработки информации и управления, а также пункты обработки информации и управления различных иерархических уровней. Подсистема передачи информации состоит из трактов передачи данных (ТПД), а подсистема внешних объектов включает как источники, так и потребители информации.

$\mathrm{K}$ качеству решения $\Phi 3$ по обработке информации и формированию управленческих решений во многих ОУ предъявляются высокие требования в части обоснованности и своевременности принятия управленческих решений. В совокупности с необходимостью обработки больших объемов информации в сжатые сроки это приводит к необходимости внедрения комплексов средств автоматизации (KCA), обеспечивающих решение ряда $\Phi 3$ в автоматизированном или автоматическом режиме.

\section{2. Вербальная постановка задачи}

Создание РИУС предполагает проектирование (модернизацию) КСА для различных иерархических уровней системы (именно в них реализуется автоматизация соответствующих ФП и ФЗ), изготовление серийных образцов $\mathrm{KCA}$, а также оснащение ими органов и объектов из состава СОИУ.

При этом, исходя из существующего порядка выполнения работ по автоматизации пунктов, органов и объектов из состава СОИУ при обосновании рационального варианта выбора системотехнических решений по созданию (развитию) СОИУ необходимо учитывать имеющиеся ограничения по выделяемым ассигнованиям на 


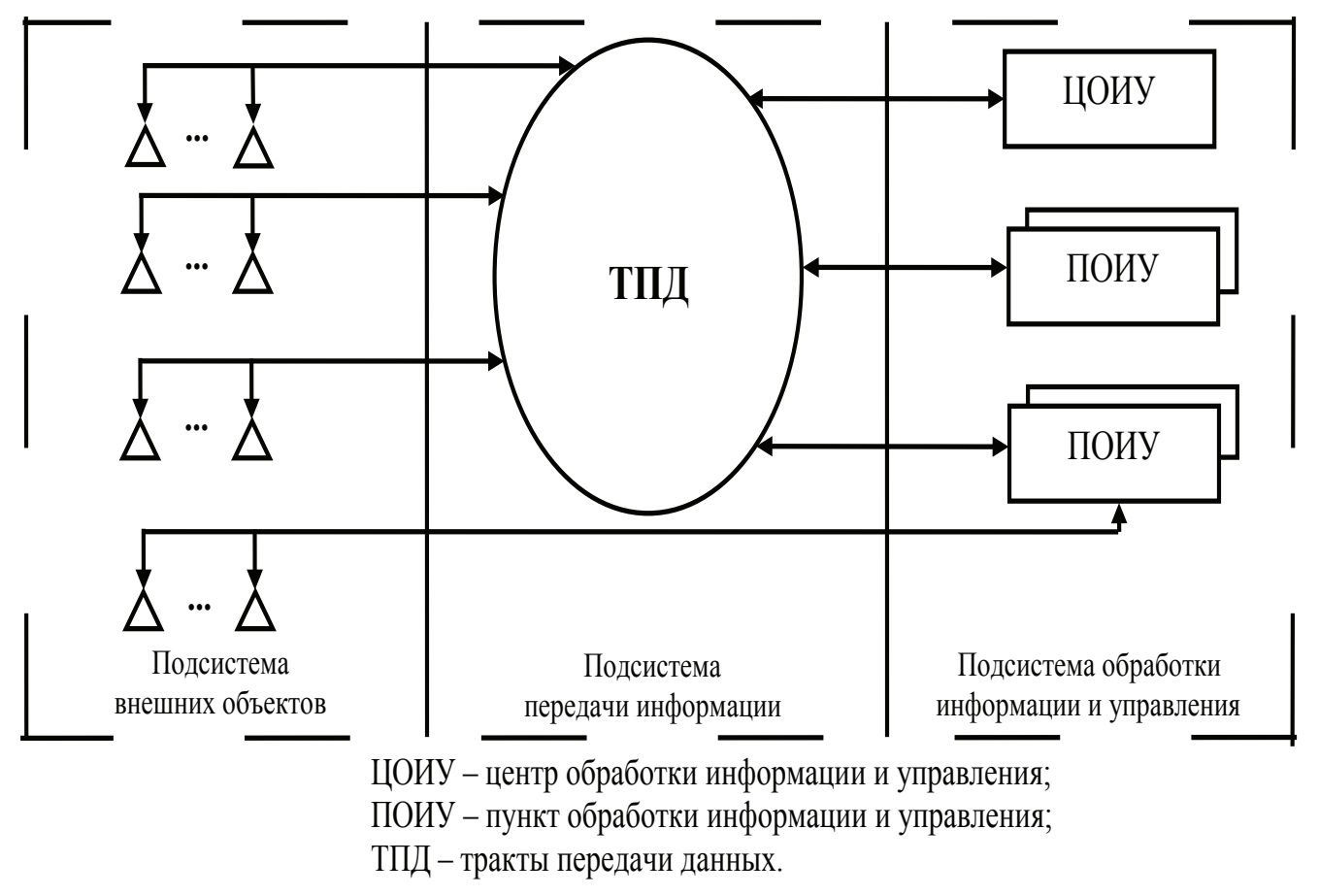

Рис. 1: Типовая схема РИУС

эксплуатацию существующих, производство серийных образцов и проектирование перспективных KCA.

Ряд теоретических исследований, связанных с вопросами выбора системотехнических, схемотехнических и организационных решений по созданию (развитию) автоматизированных систем управления, информационных систем организационного типа и оснащению ими соответствующих ОУ известны и описаны в литературе. Так, например, в [1-12] описаны некоторые подходы к оценке эффективности функционирования РИУС, их подсистем и элементов, а также ряд частных постановок задач и соответствующих методов (научно-методических подходов) по выбору ряда системотехнических, схемотехнических и организационных решений для различных этапов жизненного цикла указанного класса систем.

Тем не менее, на сегодняшний момент времени вопросы обоснования, оценки и выбора системотехнических решений по созданию (развитию) РИУС недостаточно формализованы и, в основном, основаны на интуиции и опыте разработчиков систем, комплексов и средств автоматизации. При этом, как правило, не рассматриваются вопросы сбалансированной реализации межуровневых функциональных подсистем и функциональных процессов, а оценки вероятностно-временных характеристик функционирования РИУС проводятся эмпирически только на этапах испытаний.

Ниже приводится наиболее общая формулировка задачи выбора системотехнических решений по созданию (развитию) РИУС с учетом ресурсных ограничений 
на отдельных плановых этапах, произведена ее формализация и оценка сложности решения, а также предложены общая методика и алгоритм решения.

Сущность выбора варианта решений по созданию (развитию) РИУС $[11,12]$ состоит в том, что для каждого планового этапа прогнозирования (ЭП) должны быть выбраны системотехнические решения по автоматизации ОУ за счет оснащения (дооснащения) ОУ серийными КСА (оснащение возможно в текущий момент времени) или разрабатываемыми КСА (оснащение возможно в будущем после момента окончания соответствующей опытно-конструкторской работы) с учетом допустимого (назначенного) ресурса использования КСА в составе РИУС. При этом, в процессе выбора варианта развития KCA должны быть также учтены требования по комплексной межуровневой автоматизации отдельных ФП, то есть выбор для реализации в ОУ таких $\Phi 3$, которые составляют рассматриваемый ФП.

Следует отметить, что ФП имеют различную степень важности (значимости), которая определяется степенью влияния реализуемого ФП на общую эффективность применения РИУС по целевому назначению. При этом существует подмножество таких ФП, которые должны быть обязательно реализованы в РИУС - ФП высшего приоритета.

С учетом изложенных требований в качестве целевой функции для решения рассматриваемой задачи целесообразно выбрать комплексный показатель [11, 12], отражающий степень автоматизации требуемых ФП, при необходимости выполнения ряда ФП в масштабе реального времени. Для этого ниже будем использовать два частных показателя:

1. Показатель оценки реализации ФП высшего приоритета (ФПв).

2. Показатель оценки степени автоматизации остальных ФП (ФПо).

Показатель оценки реализации ФПв (показатель 1) может быть записан как векторная дискретная булева функция, элементы которой принимают значение «1»- при реализации $\Phi П, \ll 0 »-$ в противном случае.

Показатель оценки степени автоматизации ФПо (показатель 2) может быть записан как взвешенное по важности отношение реализованных ФПо к сумме важностей всех ФПо.

При этом для показателей 1 и 2 под реализованным ФП будем понимать ФП, для которого реализованы все составляющие его $\Phi 3$ и выполняются требования к временным или вероятностно-временным характеристикам (BX, BBX) его выполнения (в случае, если такие требования для рассматриваемого ФП заданы).

В качестве исходных данных, используемых для решения рассматриваемой задачи, приняты:

1. Организационная структура РИУС, описываемая как множество ОУ и связей подчинения и взаимодействия между ними. При этом каждый ОУ может быть оснащен не более чем одним КСА определенного типа.

2. Функциональная структура РИУС, описываемая как совокупность функциональных подсистем (ФПс). Каждая из ФПс состоит из множества ФП, составными частями которых являются $\Phi 3$.

В зависимости от предъявляемых требований к своевременности выполнения ФП подразделяются на ФП реального времени и ФП нереального времени. 
Основное различие между указанными видами ФП состоит в том, что к для ФП реального времени предъявляются требования к ВХ или ВВХ длительности их выполнения, а для ФП нереального времени такие требования не предъявляются.

В зависимости от важности, которая определяется степенью влияния реализуемого ФП на общую эффективность применения РИУС по целевому назначению, все ФП подразделяются на ФПв и ФПо.

3. Типы ОУ, каждый из которых характеризуется множеством ФЗ, подлежащих реализации в $\mathrm{KCA}$.

4. Типы KCA, предназначенные для автоматизации ОУ, в зависимости от стадии жизненного цикла КСА разделяемые на действующие (находящиеся на этапе эксплуатации в ОУ), серийные (находящиеся на этапе серийных поставок) и разрабатываемые (находящиеся на этапах выполнения соответствующих научно-исследовательских или опытно-конструкторских работ).

5. Текущий вариант оснащения ОУ $\mathrm{KCA}$, в том числе включающий сведения об типах КСА, эксплуатируемых на ОУ, их остаточном ресурсе и параметрах реализуемых в КСА ФЗ.

6. Требования к ВХ и ВВХ выполнения ФП в РИУС.

7. Перечень, организационные, производственные и финансовые характеристики предприятий, которые выполняют работы или могут принимать участие в процессах разработки, производства и поставки КСА.

8. Перечень ЭП с указанием их продолжительности и объемов финансирования.

Задача решается с учетом следующих условий и допущений [11]:

- ФП в РИУС считается реализованным в том случае, когда реализованы все $\Phi 3$, составляющие данный ФП, а также удовлетворены требования к ВX и $\mathrm{BBX}$ его выполнения (если такие требования для рассматриваемого ФП заданы);

- KCA для автоматизации ОУ представляются как неделимые элементы, в связи с чем выбор отдельных схемотехнических решений из состава $\mathrm{KCA}$ для оснащения ОУ не рассматривается;

- в рамках допустимого ресурса использования КСА всех типов, а также при принятии решения по продлению срока эксплуатации КCA, надежностные характеристики реализации ФП (Ф3) остаются неизменными и удовлетворяют заданным требованиям;

- помещения (сооружения) и объекты, в которых размещены (планируется разместить) средства автоматизации из состава РИУС, удовлетворяют требованиям по массогабаритным, климатическим и энергетическим показателям для размещения соответствующих типов $\mathrm{KCA}$ (в случае, если для ряда 
помещений (сооружений) и объектов требования по массогабаритным, климатическим и энергетическим показателям не выполняются, то к таким объектам предъявляются соответствующие требования, которые должны быть реализованы до момента поставки КСА на объекты автоматизации);

- каналы и сети передачи данных удовлетворяют информационным потребностям РИУС по обмену данными для реализации всех ФП (в случае, если для ряда направлений обмена указанные информационные потребности не выполняются, то к элементам подсистемы передачи информации (объектам телекоммуникационной инфраструктуры) предъявляются соответствующие требования, которые должны быть реализованы до момента поставки КСА на объекты автоматизации);

- должностные лица ОУ имеют требуемый уровень квалификации (то есть, при выполнении автоматизируемых $\Phi 3$ и ФП время выполнения соответствующих функций управления должностными лицами ОУ не превышает заданного);

- объекты управления, которыми должна управлять РИУС, оснащены соответствующим оконечным оборудованием, обеспечивающим интеграцию КСА в контур управления без каких-либо доработок.

\section{3. Формализованная постановка задачи}

С учетом указанных исходных данных, допущений и ограничений формализованная постановка задачи формирования решений по развитию РИУС в условиях ресурсньх ограничений может быть записана в следующем виде.

На каждом ЭП необходимо определить вариант создания (развития) РИУС на основе выбора системотехнических решений $X^{*}(u), Y^{*}(u), Z^{*}(u)$, обеспечивающий максимизацию эффективности реализации ФП Э $(X(u), Y(u), Z(u))$, при обязательной реализации ФПв, заданных предельно-допустимых ВХ и ВВX реализа-

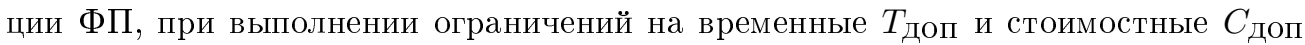
параметры процесса развития РИУС:

$$
\left\langle X^{*}(u), Y^{*}(u), Z^{*}(u)\right\rangle=\underset{X(u), Y(u), Z(u)}{\arg \max } \ni(X(u), Y(u), Z(u)),
$$

при выполнении ограничений:

$$
\begin{gathered}
\Omega(X(u), Y(u)) \cap \Omega_{\mathrm{B}}=\Omega_{\mathrm{B}} ; \\
\forall i, i \in \Omega_{\mathrm{pв} 1}: P_{i}(X(u), Y(u)) \geqslant P_{i}^{\mathrm{Tp}}(u) ; \\
\forall i, i \in \Omega_{\mathrm{pB} 2}: \tau_{i}(X(u), Y(u)) \leqslant \tau_{i}^{\mathrm{Tp}}(u) ; \\
C(X(u), Y(u), Z(u)) \leqslant C_{\text {доп }}(u) ; \\
T(X(u), Y(u), Z(u)) \leqslant T_{\text {доп }}(u) ;
\end{gathered}
$$


- $u \in\{1, \ldots U\}, U-$ количество плановых ЭП развития РИУС;

- $X(u)$ - решения по разработке $\mathrm{KCA}$;

- $Y(u)$ - решения по изготовлению $\mathrm{KCA}$ и оснащению ОУ;

- Z(u) - решения по продлению эксплуатации КСА;

- $\Omega(\mathrm{X}(\mathrm{u}), Y(u))$ - множество реализованных $Ф П ;$

- $\Omega_{\mathrm{B}}-$ множество ФПв;

- $\Omega_{\mathrm{pв} 1}$ - множество ФП реального времени, для которых предъявляются требования к $\mathrm{BBX}$ их выполнения;

- $\Omega_{\text {рв2 }}-$ множество ФП реального времени, для которых предъявляются требования к $\mathrm{BX}$ их выполнения;

- $P_{i}(X(u), Y(u))$ - вероятность своевременного выполнения $i$-го $Ф П$;

- $P_{i}^{\mathrm{Tp}}(u)$ - требуемая вероятность своевременного выполнения $i$-го $\Phi П ;$

- $\tau_{i}(X(u), Y(u))$ - среднее время выполнения $i$-го ФП;

- $\tau_{i}^{\mathrm{Tp}}(u)$ - требуемое среднее время выполнения $i$-го $\Phi П ;$

- $C(X(u), Y(u), Z(u))$ - стоимость реализации решений по созданию (развитию) РИУС;

- $C_{\text {доп }}(u)$ - финансовые ограничения на развитие РИУС;

- $T(X(u), Y(u), Z(u))$ - продолжительность выполнения работ по созданию (развитию) РИУС;

- $T_{\text {доп }}(u)$ - длительность ЭП.

\section{4. Оценка алгоритмической сложности}

Проведенный анализ алгоритмической сложности решения поставленной задачи показал, что данная задача является NP-трудной. При этом, как показано в известных работах, даже с использованием высокопроизводительной вычислительной техники, получить точное решение такой задачи методом полного перебора возможно только для относительно небольших размерностей исходных данных. Использование других точных методов дискретной оптимизации также не позволит достичь заметных преимуществ, т.к. в общем случае количество анализируемых вариантов решения сводится к количеству вариантов решения методом полного перебора.

Проведем оценку алгоритмической сложности решения задачи наиболее трудоемким точным методом - методом полного перебора. Для этого введем следующие обозначения:

- $N$ - количество ОУ (объектов автоматизации) из состава РИУС; 
- $K_{n}$ - количество типов КСА для каждого ОУ, $n \in\{1, \ldots N\}$;

- $M_{n}$ - количество ФЗ каждого ОУ.

Тогда общее количество вариантов разработки КСА, которое необходимо сформировать и оценить в процессе решения, будет рассчитываться в соответствии со следующим выражением:

$$
Q_{1}=\left(\prod_{n=1}^{N}\left(K_{n}+1\right) 2^{M_{n}}\right)^{U}
$$

Общее количество вариантов изготовления КСА и оснащения ОУ будет рассчитываться в соответствии со следующей формулой:

$$
Q_{2}=\left(\prod_{n=1}^{N}\left(K_{n}+1\right)\right)^{U}
$$

Общее количество вариантов продления срока эксплуатации КСА будет рассчитываться в соответствии со следующим выражением:

$$
Q_{3}=2^{N U}
$$

Соответственно, общее количество вариантов решения задачи будет равно произведению трех вышеуказанных выражений:

$$
Q=Q_{1} \cdot Q_{2} \cdot Q_{3}
$$

\section{5. Оценка практической возможности решения задачи точным методом}

Для оценки практической возможности решения поставленной задачи методом полного перебора зададим следующие значения исходных данных:

$U=5, N=8, K_{n}=3, M_{n}=200$.

Тогда вышеуказанные выражения примут следующие значения:

$Q_{1}=\left(4 \times 2^{200}\right)^{5 \times 8}=2^{80} \times 2^{1600}=2^{1680}$.

$Q_{2}=4^{5 \times 8}=2^{80}$.

$Q_{3}=2^{40}$.

$Q=2^{1800} \approx 7,14 \times 10^{541}$.

Следует отметить, что процессы формирования и оценки различных вариантов решения независимы и могут выполняться параллельно. Поэтому для поиска решения задачи целесообразно использовать современные подходы организации вычислительного процесса, основанные на многопоточной и многопроцессорной параллельной обработке данных. При использовании таких подходов в вычислительный процесс может быть вовлечено практически неограниченное количество вычислительных устройств, в качестве которых могут выступать как специализированные серверы, так и персональные ЭВМ. Основным ресурсом, расходуемым при таком подходе, является процессорное время, от которого монотонно и 
практически линейно (для отдельно взятого вычислительного устройства) зависит расходуемое количество электрической энергии. В связи с этим для оценки практической возможности решения поставленной задачи для указанных размерностей исходных данных рассчитаем стоимость затрачиваемой на поиск решения электрической энергии. В качестве допущения примем следующие положения:

1. Оценка одного варианта решения осуществляется за одну операцию (очевидно, что это весьма оптимистичная оценка).

2. Использование одного Вт×с электроэнергии позволяет выполнить $9,46 \times 10^{9}$ операций. Данная величина соответствует наилучшему показателю энергоэффективности по данным рейтинга Green500 [13].

Тогда для формирования и оценки $7.14 \times 10^{541}$ вариантов решений потребуется $7.55 \times 10^{531}$ Вт $\times$ с электроэнергии. При действующих на момент написания статьи тарифах для населения в 3.96 рублей за 1 кВт×ч электроэнергии, суммарная стоимость затрат на поиск решения составит $2.1 \times 10^{525}$ рублей. Данная величина в $9.65 \times 10^{511}$ раз превышает ВВП России за 2016 год, что позволяет сделать вывод о практической невозможности решения поставленной задачи методом полного перебора для заданных размерностей исходных данных.

Как было сказано выше, метод полного перебора является наиболее трудоемким точным методом поиска оптимального решения. Применение других, менее трудоемких точных методов (например, метода ветвей и границ) для задач подобного класса позволяет находить решение для больших размерностей, но, как показано в [9], тоже для весьма ограниченных размерностей исходных данньх.

\section{6. Обоснование возможности применения приближенных методов}

Рассмотрим возможность применения приближенных методов оптимизации для решения поставленной задачи.

При использовании приближенных методов, основанных на применении «жадных» алгоритмов (greedy algorithm) всегда делается выбор, который кажется самым лучшим в данный момент, т.е. производится локально оптимальный выбор в надежде, что он приведет к оптимальному решению глобальной задачи. Жадные алгоритмы не всегда приводят к оптимальному решению, но во многих задачах они дают нужный результат.

Оценку возможности применения приближенных методов решения произведем для частного случая общей поставленной задачи (1) - задачи выбора состава ФЗ для реализации в $\mathrm{KCA}$ на одном ОУ для одного ЭП без учета требований к ВВХ и $\mathrm{BX}$ их реализации [1], что соответствует заданным значениям $U=1, N=1$, $K_{1}=0$. Тогда формализованная постановка задачи примет вид:

$$
X^{*}=\underset{x}{\arg \max } \sum a_{i} x_{i}
$$

при выполнении ограничений:

$-\sum c_{i} x_{i} \leqslant C_{\text {доп }}$

$-\sum t_{i} x_{i} \leqslant T_{\text {доп, }}$ 
где

$-i \in\{1, \ldots, N\}$

- $N$ - количество задач, реализация которых возможна в $\mathrm{KCA}$;

- $X=\left\{x_{i}\right\}$ - решение по выбору задач для реализации в $\mathrm{KCA}\left(x_{i}=1\right.$, если $i$-я задача выбрана для реализации, $x_{i}=0$ - в противном случае);

- $a_{i}$ - важность реализации $i$-й задачи;

- $c_{i}$ - стоимость реализации $i$-й задачи;

- $t_{i}$ - продолжительность выполнения работ по реализации $i$-й задачи;

- $C_{\text {доп }}$ - максимально допустимая суммарная стоимость реализации задач в $\mathrm{KCA}$;

- $T_{\text {доп }}$ максимально допустимая суммарная продолжительность реализации задач в $\mathrm{KCA}$.

Для указанного частного случая поставленной задачи алгоритмическая сложность поиска решения методом полного перебора будет оцениваться по формуле $G=2^{N}$

Тогда для принятия решения по выбору задач при $N=200$ потребуется оценить $2^{200} \approx 1.6 \times 10^{60}$ вариантов решений. Используя вышеизложенный подход к оценке практической возможности решения данной задачи на основе стоимости затрачиваемых энергетических ресурсов, нетрудно подсчитать, что для поиска решения потребуется израсходовать объем электроэнергии на сумму не менее $4.71 \times 10^{43}$ рублей, что позволяет подтвердить вывод о невозможности применения метода полного перебора для решения указанного частного случая поставленной задачи при заданной размерности исходных данных.

Рассмотрим «жадные» алгоритмы решения указанной частной задачи, предложенные в [1].

1. Первый «жадный» алгоритм выбирает из вектора $a$ наибольшее значение эффективности, удовлетворяющее условию ограничения суммарной стоимости. Затем ищется следующее по величине значение эффективности и суммируется с предыдущим, если при этом соблюдается ограничение суммарной стоимости.

2. Второй «жадный» алгоритм выбирает из вектора $c$ наименьшее значение стоимости, удовлетворяющее условию ограничения суммарной стоимости. Затем ищется следующее по величине значение стоимости и суммируется с предыдущим, если при этом соблюдается ограничение суммарной стоимости.

3. Третий «жадный» алгоритм выбирает наибольшее значение отношения эффективность/стоимость. Затем ищется следующее по величине значение отношения эффективность/стоимость, эффективности и стоимости суммируются соответственно, если при этом соблюдается ограничение суммарной стоимости. 
4. Комплексный «жадный» алгоритм представляет решение задачи тремя вышеперечисленными «жадными» алгоритма и выбор решения, обеспечивающего максимальное значение целевой функции.

Для каждого из приведенных «жадных» алгоритмов был измерен коэффициент точности $W=\frac{F_{g}}{F_{a}} \times 100 \%$, где $F_{g}$ - экстремальное значение целевой функции (суммарной эффективности), полученное «жадным» алгоритмом, $F_{a}$ - экстремальное значение целевой функции (суммарной эффективности), полученное точным методом.

Для задачи с размерностью 50 в условиях случайного задания значений $a_{i}, c_{i}$,

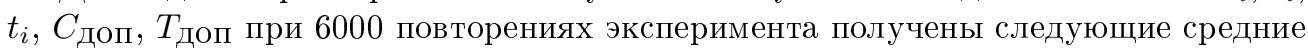
значения коэффициента точности $\mathrm{W}$, приведенные в Таблице 1.

Таблица 1: Средний коэффициент точности «жадных» методов

\begin{tabular}{|l|l|l|}
\hline Метод & $\begin{array}{l}\text { Средний коэффици- } \\
\text { ент точность } W, \%\end{array}$ & $\begin{array}{l}\text { Процент случаев, } \\
\text { при которых точ- } \\
\text { ность данного метода } \\
\text { максимальна, \% }\end{array}$ \\
\hline Первый «жадный» & 72.76 & 0.1 \\
\hline Второй «жадный» & 84.68 & 0.4 \\
\hline Третий «жадный» & 99.32 & 99.1 \\
\hline Комплексный «жадный» & 99.36 & 99.4 \\
\hline
\end{tabular}

Как показали расчеты, приближенный метод, основанный на применении «жадных» алгоритмов, обладает значительным быстродействием. Экспериментально полученное время оптимизации вторым «жадным»алгоритмом для ЭВМ с процессором AMD AthlonTM 64x2 Dual Core Processor 5000+ 2.61 ГГц и 2 Гб ОЗУ приведено в Таблице 2.

Таблица 2: Время выполнения оптимизации вторым «жадным» алгоритмом для разных размерностей задачи

\begin{tabular}{|l|l|l|l|l|}
\hline $\begin{array}{l}\text { Размерность } \\
\text { задачи }\end{array}$ & $\begin{array}{l}\text { Число повто- } \\
\text { рений экспе- } \\
\text { римента }\end{array}$ & $\begin{array}{l}\text { Среднее } \\
\text { арифметиче- } \\
\text { ское время, } \\
\text { с }\end{array}$ & $\begin{array}{l}\text { Максимальное } \\
\text { время, с }\end{array}$ & $\begin{array}{l}\text { Минимальное } \\
\text { время, с }\end{array}$ \\
\hline 100 & 100 & 0.0003 & 0.0160 & $<0.000001$ \\
\hline 1000 & 100 & 0.0203 & 0.0470 & 0.0150 \\
\hline 10000 & 100 & 1.9888 & 2.3590 & 1.9210 \\
\hline 100000 & 20 & 194.8319 & 200.2500 & 191.5940 \\
\hline
\end{tabular}

C помощью метода наименьших квадратов для второго «жадного» алгоритма была найдена аппроксимирующая функция [1] $t=0,65 \times 2^{0,000057^{N}}$.

График данной функции приведен на Рис. 2.

В идеальном случае, когда все исходные данные для решения задачи не содержат погрешности, наиболее эффективное решение дают точные методы. Если 


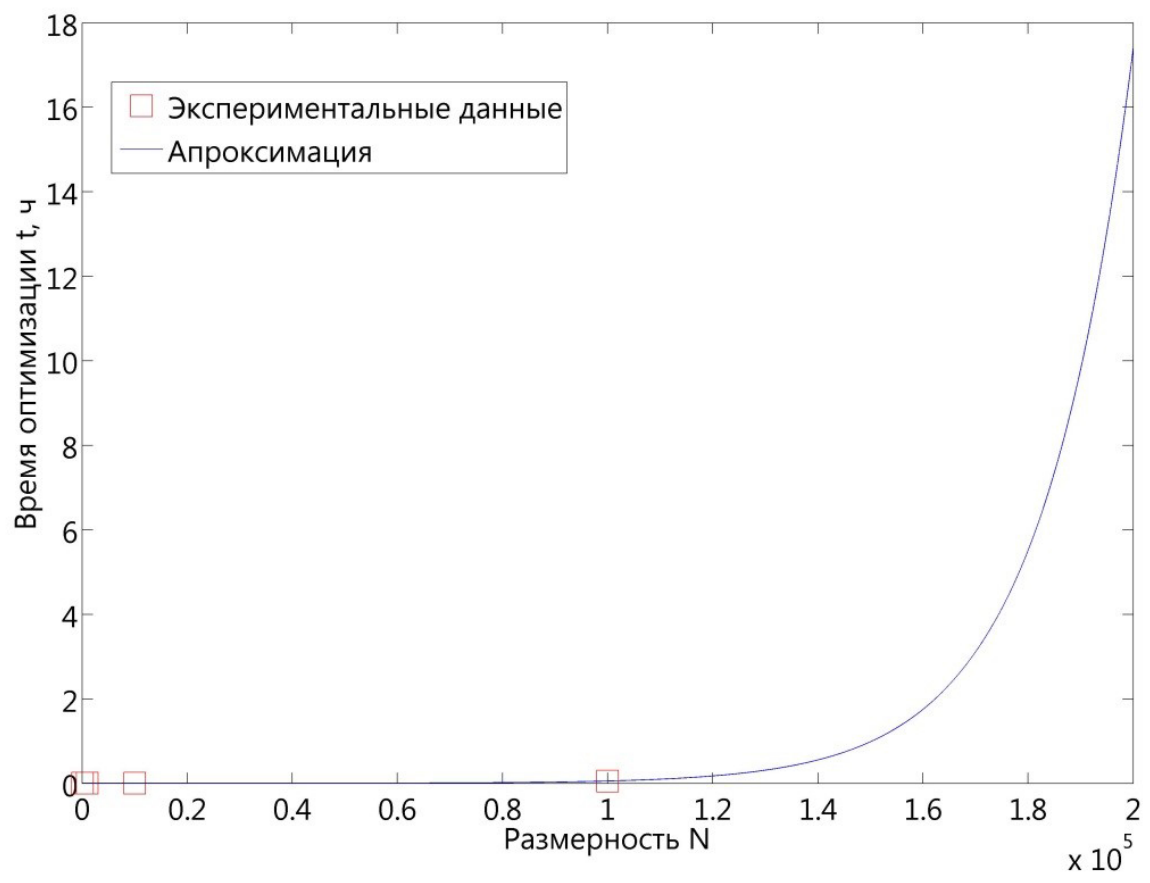

Рис. 2: Среднее время выполнения второго «жадного» алгоритма для разных размерностей задачи

исходные данные для решения задачи определены неточно (что, как правило, бывает на ранних этапах проектирования РИУС), то допустимо применять приближенные методы решения задачи оптимизации.

В [1] в ходе проведения статистического эксперимента (Рис. 3) была получена зависимость относительной эффективности решений комплексного «жадного» алгоритма при решении поставленной задачи (2), от относительной погрешности входных данных (входные данные задавались в виде случайной величина с равномерным распределением, было проведено 6000-9000 испытаний для каждой точки на Рис. 3).

Таким образом, на основании проведенных исследований можно сделать вывод, что на начальном этапе проектирования РИУС, когда входные данные для решения задач выбора системотехнических решений по созданию (развитию) РИУС определены неточно, для решения поставленной задачи целесообразно использовать комплексный «жадный» алгоритм.

В связи с этим был предложен эвристический алгоритм решения (рисунок 4), основанный на итеративно-последовательном формировании решения, начиная $\mathrm{c}$ реализации в РИУС наиболее важных ФП.

Сущность решения задачи (1) с использованием предложенного алгоритма заключается в выполнении следующих действий [12]:

1. Формирование базового варианта решения для каждого ЭП. 


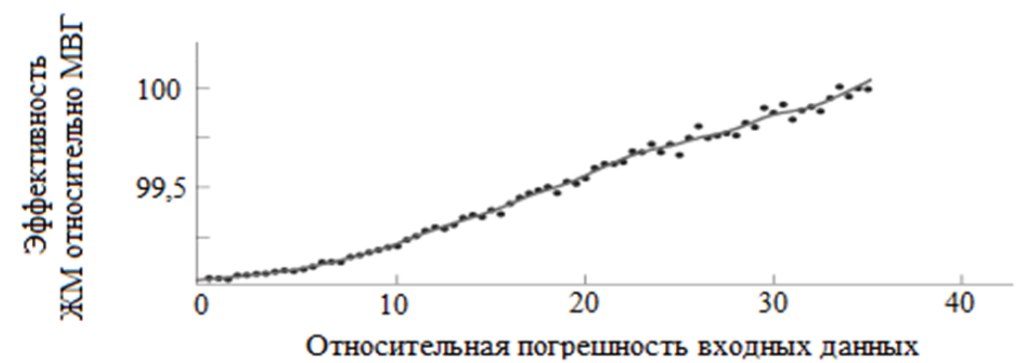

Рис. 3: Зависимость относительной эффективности решений комплексного «әнадног» алгоритма при решении задачи (2)

1.1. Последовательное рассмотрение всех ФПс в порядке убывания их важности.

1.2. Для выбранной ФПс осуществляется последовательное рассмотрение всех ФПв в порядке убывания их важности.

1.3. Для выбранного ФПв осушествляется последовательное рассмотрение всех ОУ, ФЗ которых входят в рассматриваемый ФПв, в порядке уменьшения суммарной важности $Ф 3$. ЭП.

1.4. Для выбранного ОУ осуществляется последовательное рассмотрение всех

1.5. Для выбранного ЭП осуществляются:

1.5.1. Оценка полноты реализации всех $\Phi 3$ выбранного ФПв. Если реализованы не все $\Phi 3$ выбранного ФПв, то осуществляется переход к процедуре создания решения на оснащение ОУ новым KCA на ЭП. Затем осуществляется переход к очередному ЭП (п. 1.5).

1.5.2. Оценка удовлетворения требований к своевременности решения (на основе оценки ВX и ВВХ) всех $Ф 3$ выбранного ФПв в КСА. Если требования к своевременности выполняются не для всех $\Phi 3$ выбранного ФПв, то осуществляется переход к процедуре создания решения на оснащение ОУ новым КСА на ЭП. Затем осуществляется переход к очередному ЭП (п. 1.5).

1.5.3. Оценка остаточного ресурса KCA на ОУ. Если остаточный ресурс КСА меньше продолжительности ЭП и стоимость продления эксплуатации не превышает остаточный объем финансирования ЭП, то создается решение на продление эксплуатации КСА. Затем осуществляется переход к очередному ЭП (п. 1.5).

1.6. Если рассмотрены все ЭП, то осуществляется переход к очередному ОУ (п. 1.4).

1.7. Если рассмотрены все ОУ, то осуществляется переход к очередному ФПв (п. 1.3).

1.8. Если рассмотрены все ФПв, то осуществляется переход к очередной ФПс (п. 1.2).

1.9. Если рассмотрены все ФПс, то базовый вариант решения сформирован и осуществляется переход к п. 2.

2. Формирование рационального варианта для каждого ЭП.

Выполняются действия по п. 1.1-1.9, при этом все ФПв считаются реализо- 


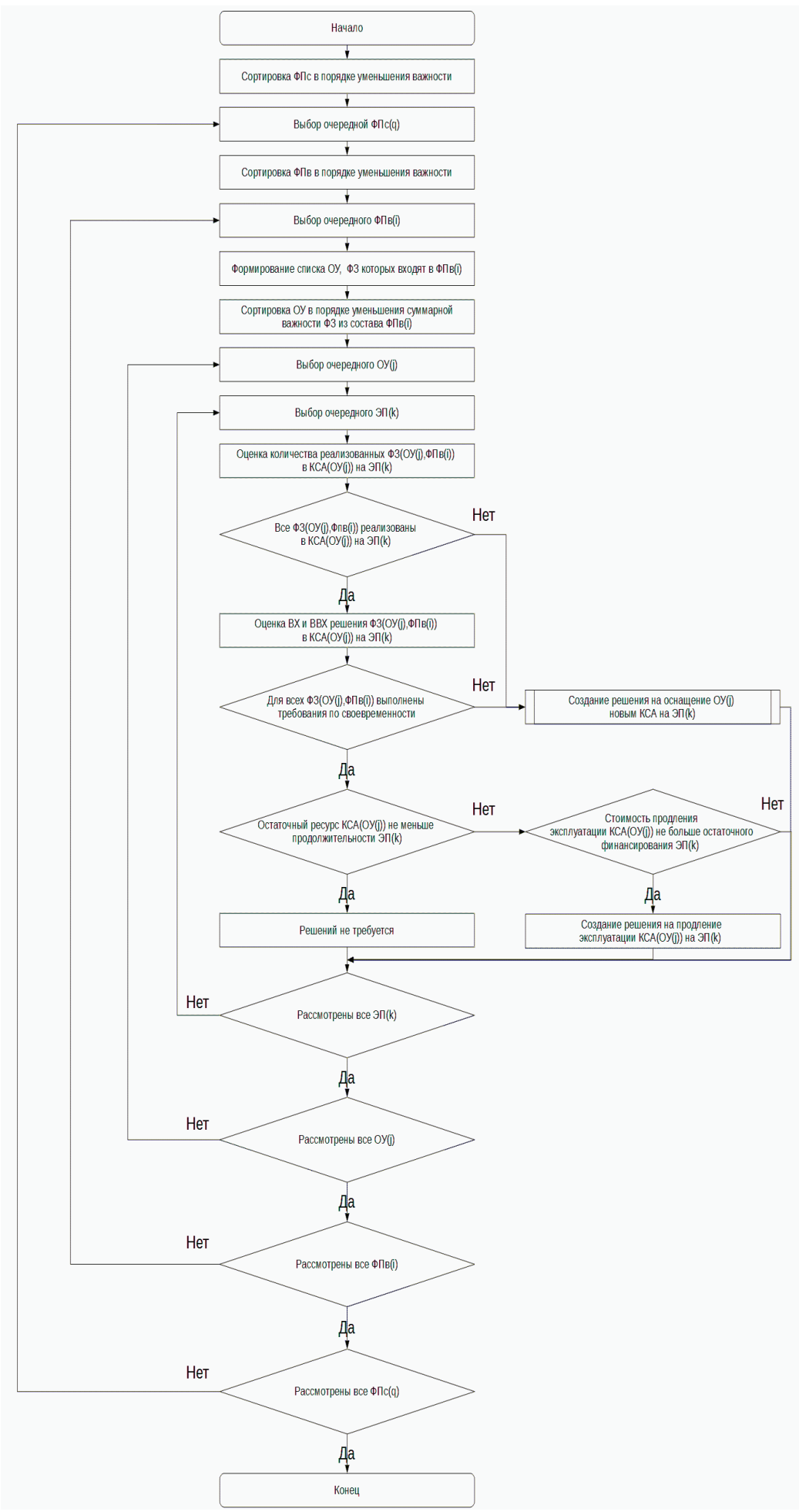

Рис. 4: Алгоритм решения задачи формирования решения по созданию (развитию) РИУС 
ванными, и последовательно (в соответствии со значением параметра важности) рассматриваются $Ф П$ не из состава ФПв, задача решается в условиях оставшихся ресурсов.

\section{Заключение}

Таким образом, в настоящей статье рассмотрены постановка задачи выбора системотехнических решений по созданию (развитию) РИУС, проведен анализ ее алгоритмической сложности и применимости различных методов ее решения, предложен эвристический алгоритм, позволяющий обосновать решения по разработке и изготовлению КСА для оснащения ОУ из состава РИУС, а также продления ресурса действующих в системе КСА.

\section{Список литературы}

[1] Лясковский В.Л. Системотехнические основы автоматизации процессов обработки информации и управления в иерархических системах военного назначения. Тверь: ВА ВKO, 2014. 244 с.

[2] Бушуев С.Н., Осадчий А.С., Фролов В.М. Теоретические основы создания информационно-технических систем. СПб.: ВАС, 1998. 382 с.

[3] Шпак В.Ф., Директоров Н.Ф., Мирошников В.И., Навойцев С.П., Наумов В.Н., Серегин А.В., Синещук Ю.И., Туровский О.М. Информационные технологии в системе управления ВМФ. СПб.: Элмор, 2005. 832 с.

[4] Липаев В.В. Системное проектирование сложных программных средств для информационных систем. М: СИНТЕГ, 2002. 268 с.

[5] Бородакий Ю.В., Боговик А.В., Курносов В.И., Карпов Е.А., Лободинский Ю.Г., Масановец В.В., Паращук И.Б. Основы теории управления в системах специального назначения / Под общей ред. Ю.В. Бородакия, В.В. Масановца. М.: Управление делами Президента Российской Федерации, 2008.

[6] Khan K.M., Zhang Y. Managing Corporate Information Systems Evolution and Maintenance. Idea Group Inc (IGI), 2005. 376 p.

[7] Лясковский В.Л., Алашеев М.А., Пильщиков Д.Е., Вакуленко А.А., Неплюев О.Н., Тикменов В.Н. Методика выбора состава задач и комплексов средств автоматизации для многоуровневой системы управления РЭС // Радиотехника. 2004. № 10. С. $75-78$.

[8] Арепин Ю.И., Допира Р.В., Смоляков А.А. Военная кибернетика: Методология создания автоматизированных систем управления техническим обеспечением. Тверь: ЗАО НИИ ЦПС, 2006. 203 с.

[9] Лясковский В.Л. Методологические основы создания (развития) и оснащения иерархических автоматизированных систем специального назначения. Тверь: BA BKO, 2010. 284 c. 
[10] Лясковский В.Л., Алашеев М.А., Морозов О.Г., Потапов В.Н. Вопросы создания интегрированных автоматизированных систем организационного управления // Инфокоммуникационные технологии. 2007. Т. 5, № 2. С. 62-64.

[11] Лясковский В.Л., Бреслер И.Б. К вопросу формализации общей задачи выбора направлений развития иерархических информационно-управляющих систем военного назначения за счет повышения степени автоматизации функциональных процессов в условиях ресурсных ограничений // Вооружение и экономика. 2016. № 4 (37). С. 36-45.

[12] Лясковский В.Л., Бреслер И.Б., Алашеев М.А. Постановка задачи формирования направлений развития автоматизированных систем организационного типа и алгоритм ее решения // Программные продукты и системы. 2017. № 2. C. $165-171$.

[13] Green500 list of the top 500 supercomputers in the world by energy efficiency [Электронный ресурс] // TOP500. URL: https://www.top500.org/green500/ (дата обращения: 22.03.2017)

\section{Образец цитирования}

Лясковский В.Л., Бреслер И.Б., Алашеев М.А. Формализация, оценка алгоритмической сложности и метод решения задачи выбора системотехнических решений по созданию (развитию) распределенных информационно-управляющих систем // Вестник ТвГУ. Серия: Прикладная математика. 2017. № 3. С. 73-91. https://doi.org/10.26456/vtpmk180

\section{Сведения об авторах}

\section{1. Лясковский Виктор Людвигович}

советник генерального директора по научной работе и инновациям АО «Научно-исследовательский институт информационных технологий».

Россия, 170100, ул. Володарского, д. З, АО «НИИИТ».

\section{2. Бреслер Игорь Борисович}

генеральный директор АО «Научно-исследовательский институт информационных технологий».

Россия, 170100, ул. Володарского, д. 3, АО «НИИИТ».

\section{3. Алашеев Михаил Александрович}

специалист научно-координационного отдела АО «Научно-исследовательский институт информационных технологий».

Россия, 170100, ул. Володарского, д. З, АО «НИИИТ». 


\title{
FORMALIZATION, ESTIMATION OF ALGORITHMIC COMPLEXITY AND A METHOD FOR SOLVING THE PROBLEM OF CHOOSING SYSTEM-TECHNICAL SOLUTIONS FOR THE CREATION (DEVELOPMENT) OF DISTRIBUTED INFORMATION-CONTROL SYSTEMS
}

\author{
Lyaskovskiy Viktor Ludvigovich \\ CEO's counselor for research and innovation, \\ JSC «Research Institute of Information Technologies». \\ Russia, 170100, Tver, 3 Volodarskogo str., JSC «RIIT» \\ Bresler Igor Borisovich \\ CEO, JSC «Research Institute of Information Technologies». \\ Russia, 170100, Tver, 3 Volodarskogo str., JSC «RIIT» \\ Alasheev Mihail Aleksandrovich \\ Specialist of Scientific Coordination department, \\ JSC «Research Institute of Information Technologies». \\ Russia, 170100, Tver, 3 Volodarskogo str., JSC «RIIT»
}

Received 24.05.2017, revised 12.07.2017.

\begin{abstract}
The article deals with the formulation of the problem of choosing system-technical solutions for the creation (development) of distributed information-control systems. Importance of the task is caused by the fact that many automated systems for information processing and management have been in operation for decades after creation, while requirements for these systems change. Therefore, one should periodically bring informationmanagement systems in accordance with new requirements. The analysis of the algorithmic complexity of the solution of the problem shows the impossibility of solving it by considering all possible variants because of the exponential dependence of the number of solutions on the size of initial data. In this regard, a heuristic algorithm based on "greedy" optimization technique was developed, which allows to significantly reduce complexity and obtain a rational solution of the problem in relatively small computational time.
\end{abstract}

Keywords: information processing and control system, design of automated control systems, life cycle of control systems, automation system.

\section{Citation}

Lyaskovskiy V.L., Bresler I.B., Alasheev M.A. Formalization, estimation of algorithmic complexity and a method for solving the problem of choosing systemtechnical solutions for the creation (development) of distributed information-control 
systems. Vestnik TvGU. Seriya: Prikladnaya Matematika [Herald of Tver State University. Series: Applied Mathematics], 2017, no. 3, pp. 73-91. (in Russian). https://doi.org/10.26456/vtpmk180

\section{References}

[1] Lyaskovskiy V.L. Sistemotehnicheskie Osnovy Avtomatizacii Processov Obrabotki Informacii $i$ Upravlenija $v$ Ierarhicheskih Sistemah Voennogo Naznachenija [System-technical Basis for Automation of Information Processing and Control Processes in Hierarchical Military Systems]. VA VKO Publ., Tver, 2014. 244 p. (in Russian)

[2] Bushuev S.N., Osadchij A.S., Frolov V.M. Teoreticheskie Osnovy Sozdanija Informacionno-Tehnicheskih Sistem [Theoretical Basis for the Creation of Information Technology Systems]. VAS Publ., SPb., 1998. 382 p. (in Russian)

[3] Shpak V.F., Direktorov N.F., Miroshnikov V.I., Navojcev S.P., Naumov V.N., Seregin A.V., Sineshhuk Ju.I., Turovskij O.M. Informacionnye Tehnologii $v$ Sisteme Upravlenija VMF [Information Technologies in the Navy Control System]. Jelmor Publ., SPb., 2005. 832 p. (in Russian)

[4] Lipaev V.V. Sistemnoe Proektirovanie Slozhnyh Programmnyh Sredstv dlja Informacionnyh Sistem [System Design of Complex Software for Information Systems]. SINTEG Publ., Moscow, 2002. 268 p. (in Russian)

[5] Borodakij Ju.V., Bogovik A.V., Kurnosov V.I., Karpov E.A., Lobodinskij Ju.G., Masanovec V.V., Parashhuk I.B. Osnovy Teorii Upravlenija $v$ Sistemah Special'nogo Naznachenija [Fundamentals of Control Theory in Special-Purpose Systems]. Ed. by Ju.V. Borodakij, V.V. Masanovts. Administration of the President of the Russian Federation, Moscow, 2008. (in Russian)

[6] Khan K.M., Zhang Y. Managing Corporate Information Systems Evolution and Maintenance. Idea Group Inc (IGI), 2005. 376 p.

[7] Lyaskovskiy V.L., Alasheev M.A., Pilshhikov D.E., Vakulenko A.A., Nepljuev O.N., Tikmenov V.N. Methods for selecting the composition of tasks and complexes of automation tools for a multi-level control system for RES. Radiotehnika [Radio Engineering], 2004, no. 10, pp. 75-78. (in Russian)

[8] Arepin Ju.I., Dopira R.V., Smoljakov A.A. Voennaja Kibernetika: Metodologija Sozdanija Avtomatizirovannyh Sistem Upravlenija Tehnicheskim Obespecheniem [Military Cybernetics: Methodology for the Creation of Automated Control Systems for Technical Support]. CJSC Research Institute CPS, Tver, 2006. 203 p. (in Russian)

[9] Lyaskovskiy V.L. Metodologicheskie Osnovy Sozdanija (Razvitija) i Osnashhenija Ierarhicheskih Avtomatizirovannyh Sistem Specialnogo Naznachenija [Methodological Basis for the Creation (Development) and Equipping of Hierarchical Automated Systems for Special Purposes]. VA VKO Publ., Tver, 2010. 284 p. (in Russian) 
[10] Lyaskovskiy V.L., Alasheev M.A., Morozov O.G., Potapov V.N. The issues of creating integrate $\mathrm{d}$ automated systems of organizational management. Infokommunikacionnye Tehnologii [Infocommunication Technologies], 2007, vol. 5(2), pp. 62-64. (in Russian)

[11] Lyaskovskiy V.L., Bresler I.B. On the issue of formalizing the general task of selecting the directions for the development of hierarchical information-control systems for military purposes by increasing the degree of automation of functional processes under conditions of resource constraints. Vooruzhenie i Ekonomika [Armament and the Economy], 2016, no. 4 (37), pp. 36-45. (in Russian)

[12] Lyaskovskiy V.L., Bresler I.B., Alasheev M.A. Statement of the problem of formation of directions of development of organizational automated systems and its solution algorithm. Programmnye Produkty $i$ Sistemy [Software \& Systems], 2017, no. 2, pp. 165-171. (in Russian)

[13] Green500 list of the top 500 supercomputers in the world by energy efficiency [Electronic resource] // TOP500. URL: https://www.top500.org/green500/ (accessed at 22.03.2017) 